Né à Montréal, André Roy est rédacteur pigiste et il intervient tout autant dans le monde de l'édition et de la critique que de l'enseignement. Il a publié plus d'une vingtaine d'ouvrages, surtout de poésie, aux Herbes Rouges (revue et éditions). Parmi ses principaux titres, on remarque N'importe qu'elle page (1973), En image de ça (1974), D'un corps à l'autre (1976), Les passions du samedi (1979), Les sept jours de la jouissance (1984), Nuits (1984), C'est encore le solitaire qui parle (1986), Les amoureux n'existent que sur la Terre (1989). Il a obtenu le Prix du Gouverneur général en 1986 pour Action writing et le Grand Prix de poésie de la Fondation Les Forges en 1987 pour L'accélérateur d'intensité. Son oeuvre est une des plus remarquables de la nouvelle poésie québécoise.

\title{
Poèmes écrits après la bataille
}

\section{Le lit du soir}

Côté corps, c'est l'espace

le grand ciel piqué d'yeux

le paysage bouge, enfin bougera

à la même vitesse que nos pensées

familières de la fin du jour

des draps anciens sur lesquels

le corps a laissé sa marque

pour tous ceux qui y viendront

souffrir

\section{Un ordre de grandeur}

Chacun de nous s'en va puis revient en se consolant qu' une science

n'ait pas arrondi tout à fait la Terre 
ni allongé l'été pour les parades

de l'amour, chacun de nous

qui se tient avec les autres

(malgré que les autres ne soient pas

toujours des choses chaudes

ni promesses ni gages ni cadeaux)

est une colonne de solitude

\section{Chemin de Croix}

La nuit suit les détails

de notre existence, reconnaît

que le coeur y est beau

parfois d une beauté ressemblant à la beauté

les émotions paraissent naturellement grandes

pour notre corps qui vagit à peine

nous sortons de notre âge

comme le silence s'échappant des pierres

dans le jardin des inconnus

le réel est crucifié

à côté de nous

\section{Le vide interieur}

Dans le livre dorment la technique

et le vertige, dort aussi tout l'air

conservé dans les cendres de notre tête

avant que les neutrons pourrissent

au centre de la planète rare

(qui aura tourné pour elle-même 
pendant quelques millions d'années)

apparaitront comme une information privilégiée

pour la vie sur la Terre

les espèces en voie de guérison

\section{Mouvement}

Tristesse qui roule vers le trou de lumière dans l'été le plus grand, celui où tu t'approchais de la promesse des choses, du gage des images et de la langue des étrangers ne te consolant pourtant pas que la science ait confirmé que la Terre tournait autour de mille soleils oubliés par Dieu

\section{Devoir}

Reste encore la neige neuve, elle rappelle que nous nous aimions, c'etait le temps des héros abandonnés sur les plateaux de lumière ignorés des dieux, ignorant leur fatigue dans le pauvre jour qui les surprenait encore vivants en train de m' aimer comme toi pour me sauver 